\title{
Properties of InGaAs/InP thermoelectric and surface bulk micromachined infrared sensors
}

\author{
Alfons Dehé and Hans L. Hartnagel \\ Institut für Hochfrequenztechnik, Technische Hochschule Darmstadt, Merckstr. 25, D-64283 Darmstadt, \\ Germany \\ Dimitris Pavlidis and Kyushik Hong \\ Department of Electrical Engineering and Computer Science, The University of Michigan, 1301 Beal \\ Avenue, Ann Arbor, Michigan 48109-2122 \\ E. Kuphal \\ Forschungszentrum der Deutschen Telekom, Am Kavalleriesand 3, 64295 Darmstadt, Germany
}

(Received 11 June 1996; accepted for publication 4 September 1996)

\begin{abstract}
We present a concept for the realization of InGaAs/InP micromachined thermoelectric sensors. The advantages of InGaAs lattice matched to InP combine perfectly for this application. The high selectivity of wet chemical etching of InP against InGaAs is ideally suited for surface bulk micromachining. Thermoelectric InGaAs sensors profit from the high thermal resistivity combined with high electrical conductivity and Seebeck effect. Thanks to the material parameters a responsivity of $257 \mathrm{~V} / \mathrm{W}$ and relative detectivity of $6.4 \times 10^{8} \mathrm{~cm} \mathrm{~Hz}^{-1 / 2} / \mathrm{W}$ are expected for infrared sensors. (C) 1996 American Institute of Physics. [S0003-6951(96)02646-0]
\end{abstract}

Long-wave, infrared thermal radiation provides useful information regarding the surface temperature of an object and allows its characterization by contactless techniques. Silicon- and polysilicon-based technology serves the purpose using bulk micromachining ${ }^{1,2}$ and offers CMOS IC compatibility. Another approach that has recently been explored is the use of $\mathrm{AlGaAs} / \mathrm{GaAs}$ micromachined sensors that are compatible with MESFET technology. ${ }^{3}$ We report here a novel possibility for realizing high performance sensors using InP-based III-V technology. This approach provides the possibility of high performance operation while combining the advantages of simple fabrication procedures due to the highly selective etching properties of $\mathrm{InP} / \mathrm{InGaAs}$.

The principle of operation of thermal infrared sensors is based on the conversion of infrared radiation into heat. To measure this heat quantity it is necessary to monitor the temperature increase of the radiation absorber. Hence, thermal isolation is required for enhanced temperature differences and the resulting changes with respect to the ambient can be measured with the thermoelectric effect. Thermal isolation can be achieved by the fabrication of thin floating membranes that carry the absorber. The supporting arms of the floating membrane itself can be the thermocouples-similar to the free-standing thermopile concept realized in AlGaAs. ${ }^{4}$ This system is operational at room temperature and in principle at any other temperature. One needs, however, to ensure that the temperature of the object to be measured is well above the sensor temperature so that the resulting signal can be detected. The absorber is ideally a blackbody providing the advantage of broadband response. In terms of wavelength such a sensor can detect wavelengths only below $10 \mu \mathrm{m}$ when its temperature is $300 \mathrm{~K}$ (Wien's law).

The material parameter and design requirements can be derived by considering the measurement principle. ${ }^{3}$ The key material parameters are the thermal resistivity $W$ of the supporting bridges, the Seebeck coefficient $S$, and electrical re- sistivity $\rho$ of the thermopile assuming that the thermopile and supporting bridge are one and the same.

A major motivation to introduce a new material system such as InGaAs for the fabrication of thermoelastic sensors is the fact that it offers extremely high thermal resistivity. The thermal resistivity of InGaAs, AlGaAs, and polysilicon have been reported ${ }^{5,6}$ and their maximum values result from alloydisorder scattering. In the case of $x=0.5$ the thermal resistivity of $\operatorname{In}_{x} \mathrm{Ga}_{1-x}$ As is about $0.23 \mathrm{~km} / \mathrm{W}$ which is about an order of magnitude higher compared to the binary compositions InP and InAs and polysilicon, the commonly used material for thermoelectric sensors. Moreover, it is 2.5 times higher compared to the maximum value of AlGaAs. Since only $\mathrm{In}_{0.53} \mathrm{Ga}_{0.47}$ As can be epitaxially grown lattice matched to InP, the maximum thermal resistivity is an advantage that is offered without the need of optimization.

The $\operatorname{In}_{0.53} \mathrm{Ga}_{0.47}$ As Seebeck coefficient value will be considered next. This can be estimated on the basis of Seebeck coefficients of $\mathrm{GaAs}^{7}$ and InAs. ${ }^{8}$ Comparing, for example, materials with same doping level of about $n=4.5$ $\times 10^{16} \mathrm{~cm}^{-3}$, the Seebeck coefficient is about $-350 \mu \mathrm{V} / \mathrm{K}$ for both materials. In contrast to AlGaAs, the $\Gamma$ valley of $\mathrm{In}_{x} \mathrm{Ga}_{1-x} \mathrm{As}$ is the conduction band for the entire composition range $x$. Since the calculation of the Seebeck coefficient depends mainly on the effective density of states of the conduction band, no large changes are expected to occur with doping for the Seebeck coefficient of $\mathrm{In}_{0.53} \mathrm{Ga}_{0.47} \mathrm{As}$. The evaluation of the Seebeck coefficient is therefore done using the formalism of Ref. 7 and the material parameters of Ref. 9.

The Seebeck coefficients were calculated for $n$ - and $p$-doped $\mathrm{In}_{0.53} \mathrm{Ga}_{0.47} \mathrm{As}, \mathrm{GaAs}, \mathrm{Al}_{0.3} \mathrm{Ga}_{0.7} \mathrm{As}$, and compared to the literature data of polysilicon ${ }^{10}$ as shown in Fig. 1. It changes sign for different charge carriers and is defined negative for electron transport and positive for hole transport. This feature can be used to combine $n-\mathrm{In}_{0.53} \mathrm{Ga}_{0.47} \mathrm{As}$ and $p-\mathrm{In}_{0.53} \mathrm{Ga}_{0.47}$ As to integrated $p / n$ thermocouples where 


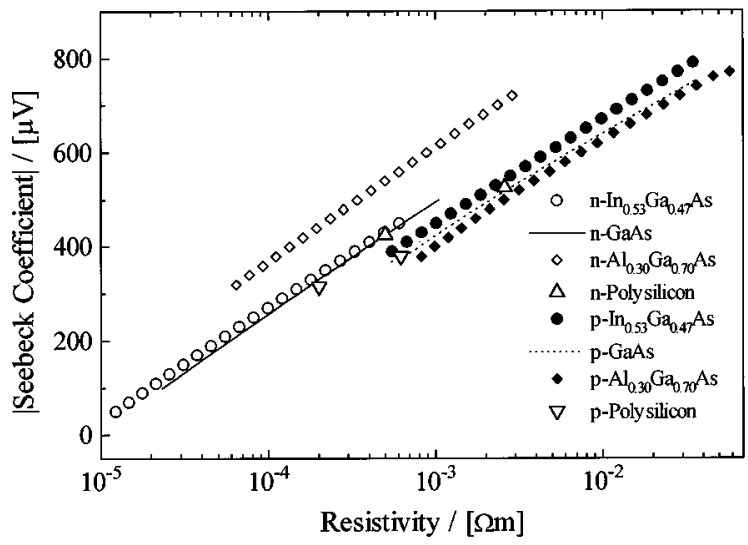

FIG. 1. Calculated Seebeck coefficients of $n$ - and $p$-doped $\operatorname{In}_{0.53} \mathrm{Ga}_{0.47} \mathrm{As}$ in comparison to $\mathrm{GaAs}, \mathrm{Al}_{0.3} \mathrm{Ga}_{0.7} \mathrm{As}$ (see Ref. 4), and polysilicon (see Ref. $10)$.

the sign of the Seebeck coefficients leads to increased sensor signal. The Seebeck effect increases with increasing resistivity due to decreasing carrier concentration. The calculations indicate no large differences between the different materials though the values for $\operatorname{In}_{0.53} \mathrm{Ga}_{0.47} \mathrm{As}$ are always slightly higher compared to $\mathrm{GaAs}, p-\mathrm{Al}_{0.3} \mathrm{Ga}_{0.7} \mathrm{As}$, and polysilicon. Only the Seebeck coefficient of $n-\mathrm{Al}_{0.3} \mathrm{Ga}_{0.7} \mathrm{As}$ is significantly higher than any of these materials. This was explained by Hava and Hunsberger ${ }^{7}$ by the fact that its composition is close to the crossover point from direct to indirect band-gap material.

The performance of thermoelectric sensors is dependent on the internal resistance of the thermocouple since Johnson noise $\left(\left(4 k_{B} T R_{\mathrm{el}} \Delta f\right)^{1 / 2}\right.$, with $k_{B}$ the Boltzmann constant, $T$ the temperature, $R_{\mathrm{el}}$ the electrical resistance of the thermopile, and $\Delta f$ the bandwidth) determines the noise equivalent power $\left[\mathrm{NEP}=\left(4 k_{B} T R_{\mathrm{el}} \Delta f\right)^{1 / 2} / R\right.$, with $R$ being the responsivity]. Hence, low electrical resistivity is required but this implies reduced Seebeck coefficient. Since InGaAs offers high mobility ${ }^{11}$ this tradeoff is relaxed compared to low mobility materials.

The power conversion factor $Z=S^{2} W / \rho$ is the figure of merit to compare different thermoelectric materials. A maximum power conversion factor is favorable for optimum op-

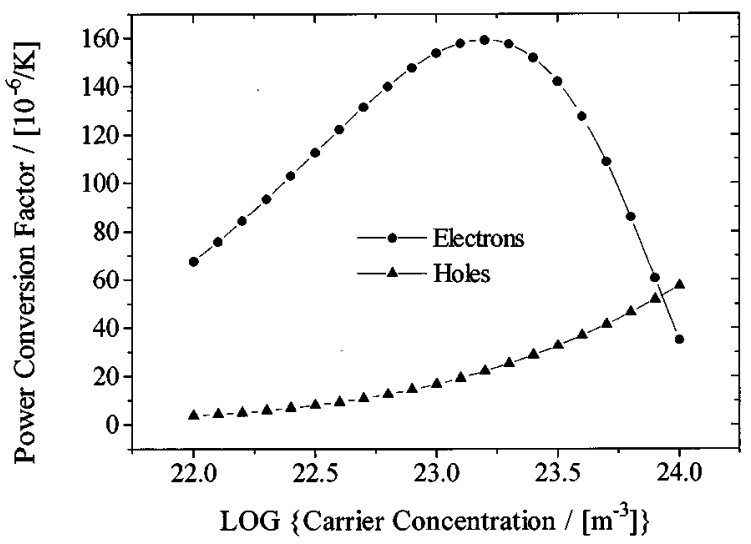

FIG. 2. Power conversion factor of $\operatorname{In}_{0.53} \mathrm{Ga}_{0.47} \mathrm{As}$ depending on electron and hole concentration.

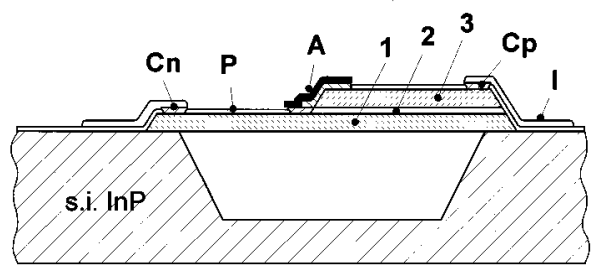

FIG. 3. Proposed structure of a free-standing $p / n$-InGaAs thermocouple bridge. The epitaxial layers are: (1) $n$-InGaAs, (2) undoped InP, (3) $p$-InGaAs. The abbreviations are: $A$ absorber, $C n / C p$ Ohmic contact to $n / p$-InGaAs, $I$ interconnect metallization, and $P$ passivation.

eration of the sensor. As Fig. 2 shows $Z$ varies with the doping level and with the charge carrier type. The power conversion shows maximum values of $160 \times 10^{-6} / \mathrm{K}$ for electron transport at doping levels around $2 \times 10^{17} \mathrm{~cm}^{-3}$. $p$-doped InGaAs is also shown to have reasonable power conversion factors for high doping level. By way of comparison the values for polysilicon used in CMOS IC is about $60 \times 10^{-6} / \mathrm{K}^{10}$ Consequently, the combination of $p$ - and $n$-InGaAs for thermoelectric devices is interesting for high performance thermoelectric sensors.

The technological advantages of the InGaAs/InP material system arise from the fact that both materials can be etched selectively against each other. This simplifies the technology for the sensor fabrication, as well as the micromachining procedure necessary for floating membrane definition.

Figure 3 shows a possible cross section of the technology along a bridge type $p / n$ thermocouple that supports an infrared absorbing area $A$ at the hot junction of the thermocouple. The epitaxial layers can be grown with standard MOCVD procedures: the first layer is a $n$-InGaAs layer ( $1 \mu \mathrm{m}, n=10^{17} \mathrm{~cm}^{-3}$ ) used for the $n$ part of the thermocouple; layer 2 consists of undoped $\operatorname{InP}(0.1 \mu \mathrm{m})$ that is used as an electrical insulation between layer 1 and layer 3; the third layer consists of $p$-InGaAs $\left(1 \mu \mathrm{m}, p=10^{19} \mathrm{~cm}^{-3}\right)$ that is used for the $p$ part of the thermocouple. Successive selective etching can be applied to form the thermocouple mesas. Due to the high doping level and low band gap of the material the Ohmic contacts $(C n)$ for $n$-InGaAs and $(C p)$ for $p$-InGaAs have very low contact resistance. To increase the output signal several thermocouples can be connected into series. After surface passivation $(P)$ with $\mathrm{SiO}_{2}$ an interconnect metallization $(I)$ is deposited. The hot junction of the thermocouple is deposited with a Gold-Black layer exhibiting high absorption coefficient near 1 and flat response to wavelength. In a final step the InP underneath the thermocouples has to be removed for thermal isolation purpose. When InP is etched $\mathrm{HCl}: \mathrm{H}_{3} \mathrm{PO}_{4}=1: 1$ can be used which offers high etching rate and extremely high selectivity with respect to InGaAs. Therefore, the fabrication of free-standing cantilevers, bridges, and floating membranes can be performed from the front side of the wafer.

The thermocouple works as follows: The absorbed heat is dissipated along the two thermocouple legs to the heat sink represented by the substrate. A temperature difference is the result. Underneath the absorbing layer $A$ the Ohmic contacts of $n$ and $p$ part of the thermocouple make electrical contact. Hence, a positive and negative thermovoltage is generated 
between absorber $A$ and the Ohmic contacts $C p$ and $C n$ on the heat sink, respectively.

Based on heat conduction estimates along the thermocouple material and heat losses via radiation we have next calculated the responsivity $R\left(R=\alpha\left(S_{p}-S_{n}\right) R_{\text {th }}\right.$, with $\alpha$ the absorption coefficient, $S_{p / n}$ the Seebeck coefficients of $p$ - and $n$-InGaAs, and $R_{\text {th }}$ the thermal resistance of the thermocouple) and relative detectivity $D^{*}\left(D^{*}=A^{1 / 2} / \mathrm{NEP}\right.$, with $A$ the absorber area) for the following sensor geometry: width of bridge $20 \mu \mathrm{m}$, length of $n$ part $300 \mu \mathrm{m}$, length of $p$ part $700 \mu \mathrm{m}$, and total absorbing area of $A=0.42 \mathrm{~mm}^{2}$. If this sensor is operated in vacuum, no thermal leakage against atmosphere is possible and maximum performance is achieved. The expected responsivity of such a sensor is $257 \mathrm{~V} / \mathrm{W}$ and its relative detectivity is $6.4 \times 10^{8}$ $\mathrm{cm} \mathrm{Hz}{ }^{-1 / 2} / \mathrm{W}$. These values are very promising since they compare with the best reported values for free-standing AlGaAs thermocouple infrared sensors ${ }^{4}$ while much simpler technology can be used.

In conclusion, the potential of the $\mathrm{InGaAs} / \mathrm{InP}$ material system is shown as applied to thermoelectric infrared sensors. The InGaAs material parameters dictating the sensor performance are presented and show advantages for the proposed sensor. In addition to material parameters, the fabrication of high performance micromachined sensors using this technology can be considerably simplified. Besides the ap- plication in remote temperature control, the same technology can be applied to integrated sensor arrays serving as infrared image sensors. Integration with InP integrated electronics such as HEMT and HBT or optoelectronic components such as diode lasers (beam control) may also be envisaged and opens up new fields of application.

The authors thankfully acknowledge the financial support of this work by the "Deutsche Forschungsgemeinschaft', (SFB241), by Alexander von Humbolt Fellowship and URI/ARO (Contract No. DAAL03-92-G-0109).

${ }^{1}$ J. Schieferdecker, R. Quad, E. Holzenkämpfer, and M. Schulze, Sens. Actuators A 46-47, 422 (1995).

${ }^{2}$ R. Leggenhager, H. Baltes, and T. Ebel, Sens. Actuators A 37-38, 216 (1993)

${ }^{3}$ A. Dehé, K. Fricke, and H. L. Hartnagel, Sens. Actuators A 46-47, 432 (1995).

${ }^{4}$ A. Dehé and H. L. Hartnagel, IEEE Trans. Electron Devices 43 (1996) (to be published).

${ }^{5}$ S. Adachi, J. Appl. Phys. 54, 1844 (1983).

${ }^{6}$ F. Völklein and H. Baltes, J. Microelectromechanical Syst. 1, 193 (1992).

${ }^{7}$ S. Hava and R. Hunsberger, J. Appl. Phys. 57, 5330 (1985).

${ }^{8}$ Semiconductors, Landolt-Börnstein (Springer, Berlin, 1984), Vol. 17, p. 578.

${ }^{9}$ W. Kowalsky, Dissertation at the Technische Universität CaroloWilhelmina, Braunschweig, Germany, 1985.

${ }^{10}$ F. Völklein and H. Baltes, Sens. Mater. 3, 325 (1992).

${ }^{11}$ E. Kuphal and D. Fritzsche, J. Electron. Mater. 12, 743 (1983). 